\title{
Laparoscopic spleen-preserving distal pancreatectomy for a solid-cystic intraabdominal desmoid tumor at a gastro- pancreatic lesion: a case report
}

Keishi Sugimachi ${ }^{1 *}$ D, Tomohiro Iguchi ${ }^{1}$, Mitsuhiko Ohta ${ }^{2}$, Yohei Mano ${ }^{1}$, Terumasa Hisano ${ }^{3}$, Ryohei Yokoyama ${ }^{4}$, Kenichi Taguchi ${ }^{5}$, Masahiko Ikebe ${ }^{2}$, Masaru Morita ${ }^{2}$ and Yasushi Toh ${ }^{2}$

\begin{abstract}
Background: We report a case of an intraabdominal desmoid tumor that occurred at a gastro-pancreatic lesion with spontaneous cystic features, and present the successful laparoscopic resection of the tumor.

Case presentation: A 20-mm retroperitoneal cystic mass with a solid component was found adjacent to the stomach and pancreatic body in a 52-year-old woman with no history of familial adenomatous polyposis. Laparoscopic spleen-preserving distal pancreatectomy with wedge resection of the stomach was performed, and complete resection was achieved without intraoperative and postoperative complications. Histopathological examination by immunohistochemistry enabled diagnosis of a desmoid tumor that had originated from the stomach and invaded the pancreatic parenchyma with a spontaneous cystic change. We herein report an extremely rare case of an intraabdominal desmoid tumor with a spontaneous cystic change.

Conclusion: Regardless of its rarity, desmoid tumor should be included in the preoperative differential diagnosis of a cystic intraabdominal mass, and laparoscopic function-preserving surgery may be an optimal treatment option.
\end{abstract}

Keywords: Desmoid tumor, Cyst, Spleen-preserving distal pancreatectomy, Case report

\section{Background}

Desmoid tumors (also called aggressive fibromatosis, deep musculoaponeurotic fibromatosis) are locally aggressive tumors with no potential for metastasis or dedifferentiation. Radiologically, they are usually nonspecific, slow-growing solid masses that are difficult to distinguish from other soft tissue tumors, but they rarely show cystic lesions. Sporadic desmoid tumors can occur at any site in the body, and anatomic locations are classified into 3 regions: trunk/extremity, abdominal wall, and intraabdominal. Complete surgical resection is the only curative option, but intraabdominal desmoid tumors are often unresectable, because they often infiltrate the mesentery. Here, we report a case of an intraabdominal

\footnotetext{
* Correspondence: ksugi@surg2.med.kyushu-u.ac.jp

${ }^{1}$ Department of Hepatobiliary-Pancreatic Surgery, National Hospital Organization Kyushu Cancer Center, 3-1-1 Notame, Minami-ku, Fukuoka 811-1395, Japan

Full list of author information is available at the end of the article
}

desmoid tumor that occurred at a gastro-pancreatic lesion with cystic features, and presents the successful laparoscopic resection of the tumor.

\section{Case presentation}

An intraabdominal cystic mass was incidentally detected between the stomach and the pancreas in a 52-year-old woman undergoing postoperative follow-up computed tomography (CT) for chondrosarcoma of a rib. Her family history was unremarkable. Blood counts and serum biochemistry results were within normal limits. An abdominal CT scan revealed a $20 \times 18-\mathrm{mm}$ well-defined cystic mass with a solid component (Fig. 1a,b). The tumor was adjacent to the stomach and the pancreas body, but there was no sign of invasive growth to the organs. MRI showed a cystic mass with high intensity on T2-weighted images and low intensity on T1-weighted images. The lesion showed no sign of diffusion restriction by diffusion- 
a

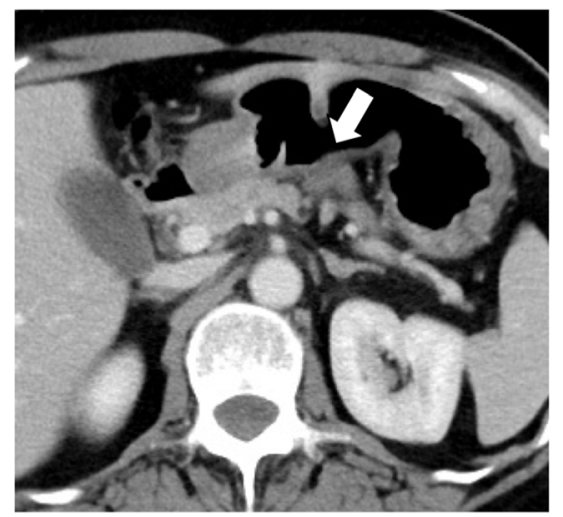

C

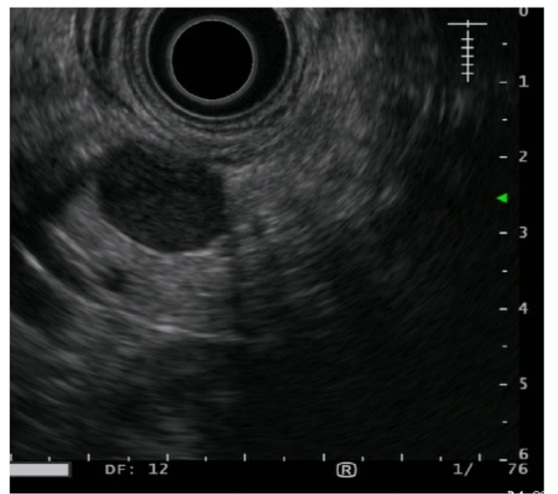

b

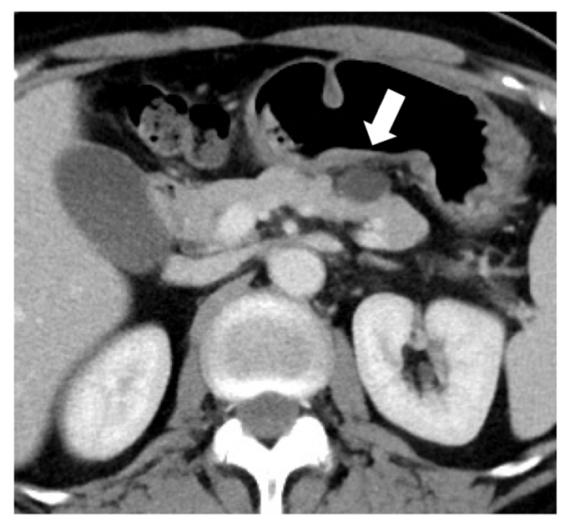

d

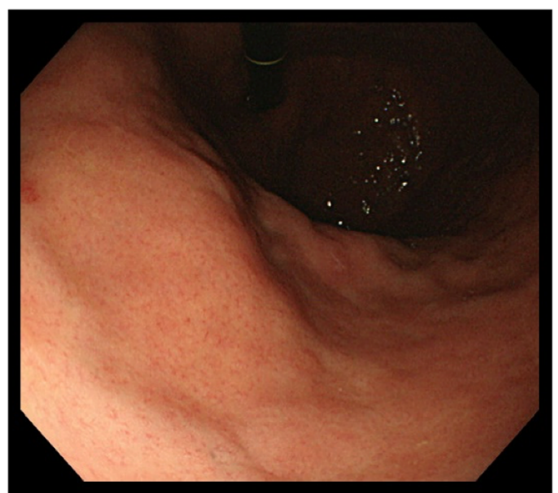

Fig. 1 Preoperative images. a, b CT image showing a well-defined round, 20-mm cystic mass with a rim of soft tissue in the gastro-pancreatic region. The tumor is dense, adjacent to the gastric wall and pancreatic parenchyma. Slight enhancement of the solid component is seen after intravenous administration of contrast material. Arrows indicate the tumor. c Endoscopic ultrasound image showing a well-defined cystic mass. $\mathbf{d}$ Gastric endoscopy showed no abnormal findings of the gastric mucosa

weighed MRI. The solid component of the cystic wall was gradually enhanced in the late phase. Subsequent endoscopic ultrasonography (EUS) showed an $18 \times 13 \mathrm{~mm}$ round cystic mass, with a heterogeneous nodular lesion at the side of the serosa of the stomach (Fig. 1c). Esophagogastric endoscopy showed no local lesion on the mucosal surface of the stomach (Fig. 1d). An intraabdominal tumor was suspected, but it was difficult to determine the specific diagnosis.

Laparoscopic resection via 5 trocars was performed with the patient in the supine position. After the gastrocolic ligament was cut, the omental bursa was opened widely. The tumor was confirmed at the superior border of the pancreas body, and adhered to the posterior wall of the stomach (Fig. 2a). With careful dissection, the left gastric artery and vein were isolated and preserved. The tumor was cut from the stomach by wedge resection of the gastric wall using a linear stapler (Fig. 2b). Then, we performed a spleen-preserving distal pancreatectomy. The pancreas body-tail was carefully mobilized and separated from the splenic artery and vein by dissecting the small vessels. The pancreas was dissected at the right side of the tumor using a linear stapler (Fig. 2c). We performed a complete laparoscopic excision, and the spleen and splenic vessels were completely preserved (Fig. 2d). The operative time was $279 \mathrm{~min}$, and intraoperative blood loss was $15 \mathrm{~mL}$. The postoperative course was uneventful, and the patient was discharged healthy.

Macroscopic examination of the resected specimen revealed a capsulated cystic mass measuring approximately $20 \mathrm{~mm}$ with serous content. The whitish solid nodule containing the cystic lesion existed at the wall of the stomach (Fig. 3a). Microscopic examination demonstrated the distribution of spindle tumor cells, partly arranged in a fascicular fashion and accompanied by a collagenous stroma (Fig. 3b, c). Mitotic figures were rarely seen. The tumor cells were attached to the muscular layer of the gastric wall (Fig. 3b). A benign cystic lesion was also observed, but its origin was not determined. Immunohistochemically, the spindle cells were diffusely positive for $\beta$-catenin protein in the nucleus (Fig. 3d), but negative for the $\alpha$ SMA, desmin, S-100, c-kit, and CD34 proteins. These findings were consistent with the diagnosis of intraabdominal desmoid tumor.

\section{Discussion and conclusions}

Desmoid tumors are rare, and their estimated incidence in the general population is $2-4$ per million per year [1]. 

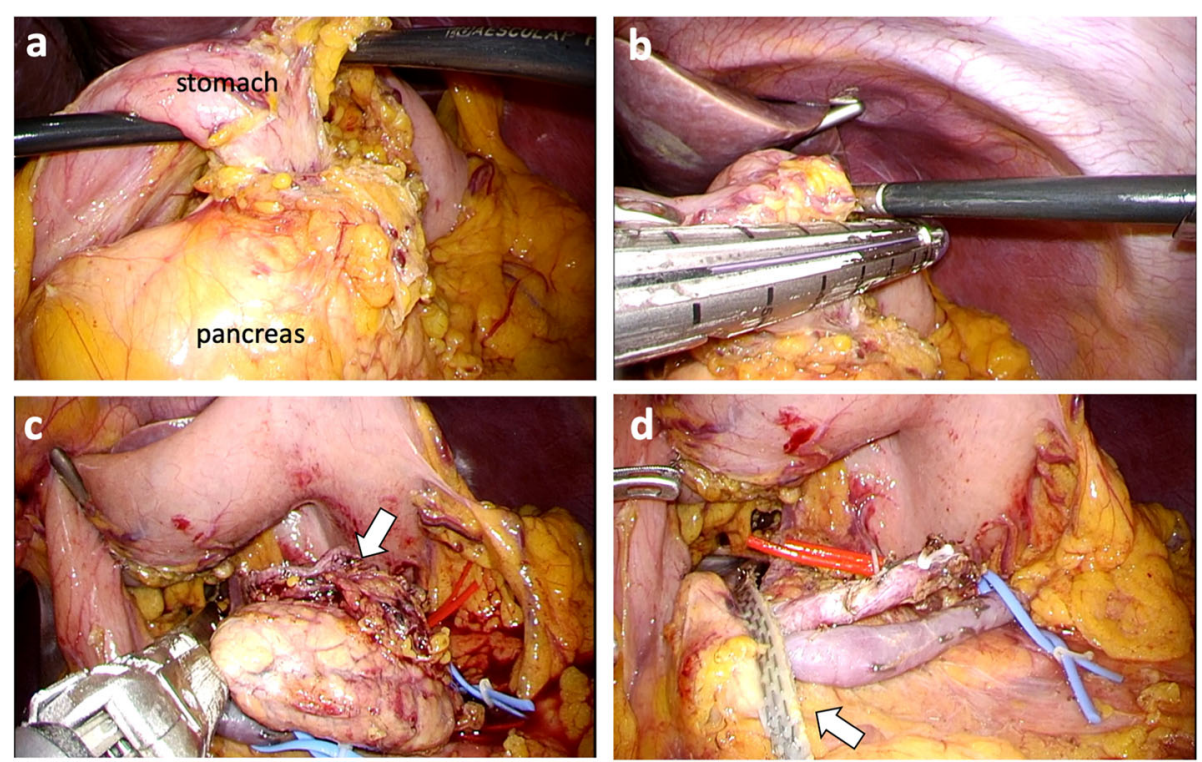

Fig. 2 Intraoperative findings. a The tumor was located in the gastro-pancreatic region and locally invaded the gastric wall and the pancreatic parenchyma. The arrow indicates the tumor. $\mathbf{b}$ Wedge resection of the stomach was performed using a linear stapler. $\mathbf{c}$ The pancreatic parenchyma was transected using a linear stapler. The arrow indicates the tumor. $\mathbf{d}$ The splenic artery (red tape) and splenic vein (blue tape) were preserved. The arrow indicates pancreatic stump
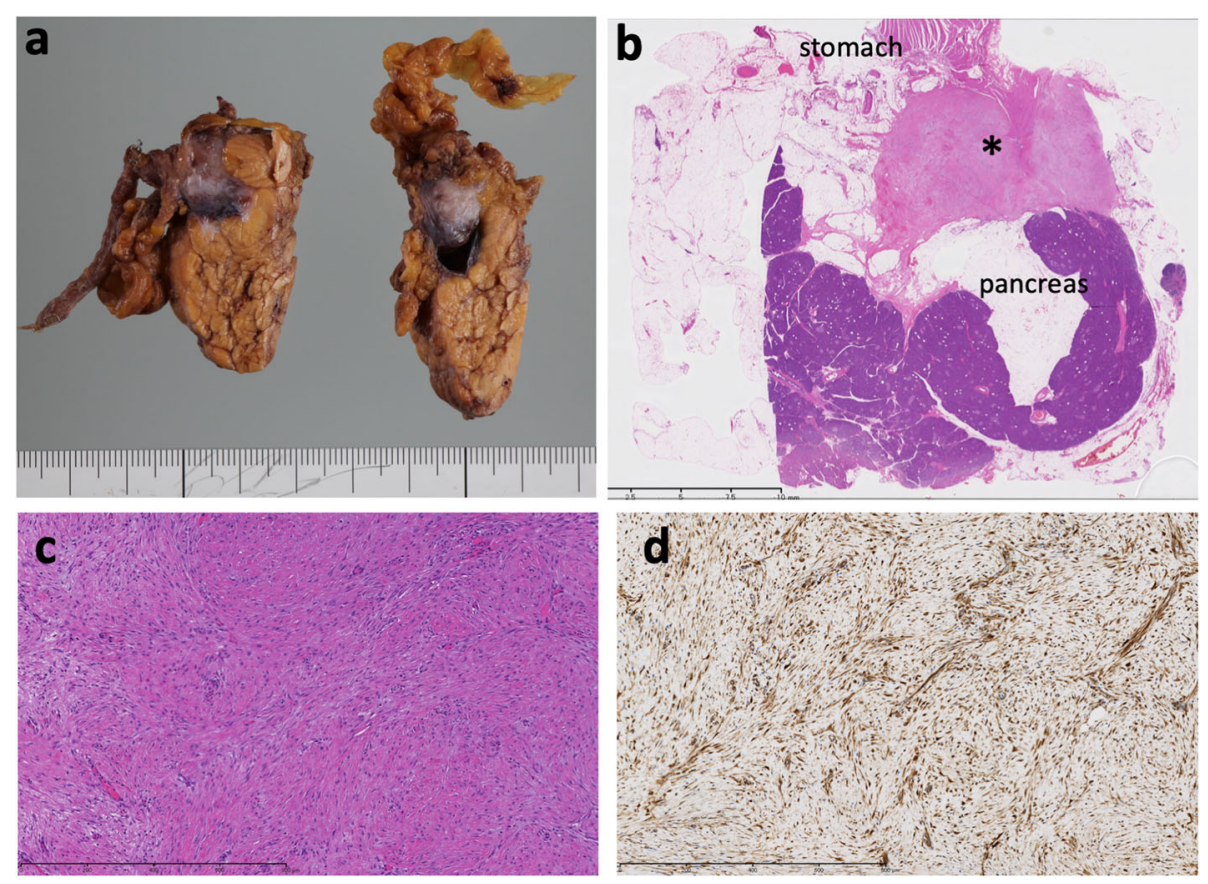

Fig. 3 Macroscopic and microscopic findings. a The cut surface of the excised solid-cystic mass showing light tan glistening tissue. The arrow indicates the tumor. $\mathbf{b}$ A lower power view shows that the tumor cells (asterisk) originated from the gastric wall and attached to the pancreatic parenchyma without invasion (hematoxylin and eosin staining; original magnification $\times 40$ ). c Histopathological findings show uniform cord-like proliferation of spindle-shaped cells with minimal atypia, and the intercellular spaces are filled with thick bundles of collagen fibers (hematoxylin and eosin staining; original magnification $\times 100$ ). $\mathbf{d}$ Immunohistochemically, the spindle cells are diffusely and strongly positive for nuclear $\beta$ catenin protein. $(\times 100)$ 
Table 1 Reported 8 cases of spontaneous cystic desmoid tumor

\begin{tabular}{llllll}
\hline No & Primary site & First author & Year & Journal & Reference \\
\hline 1 & Mesentery (2 cases) & Ko, SF & 2006 & Ultrasound Med Biol & {$[5]$} \\
2 & Pancreas & Amiot, A & 2008 & JOP & {$[6]$} \\
3 & Mesentery & Tan, CH & 2010 & Br J Radiol & {$[7]$} \\
4 & Xu, B & 2013 & World J Gastroenterol & {$[8]$} \\
5 & Pancreas & Mourra, N & 2015 & Gastroenterology & {$[9]$} \\
6 & Pancreas & Patel, HD & 2017 & ACG Case Rep J & {$[10]$} \\
7 & Pancreas & Lee, KC & 2018 & BMC Med Imaging & {$[11]$} \\
\hline
\end{tabular}

Individuals between the ages of 15 and 60 are most commonly affected, and most desmoid tumors arise sporadically; however, between 5 and 15\% are associated with familial adenomatous polyposis (FAP, Gardner syndrome) caused by mutations in the APC (adenomatous polyposis coli) gene [2]. The risk of developing a desmoid tumor in a patient with FAP is 852 times that of the general population [2]. Intraabdominal desmoid tumors are usually asymptomatic and therefore are often found incidentally. Furthermore, lack of typical imaging features makes preoperative diagnosis of desmoid tumors very difficult. Typically, imaging studies show a well-defined solid mass with homogenous enhancement and absence of intraabdominal necrosis or degeneration, which reflects the highly collagenous stroma of the tumor [3].

Desmoid tumors potentially show cystic changes with medical treatment or abscess formation [4], but a spontaneous cystic change, as described in this case, is extremely rare, with only eight cases reported in the literature (Table 1) [5-11]. Spontaneous cystic changes could be caused by regression of the tumor associated with the withdrawal of estrogenic stimulation, infection or secondary infarction [11]. In the literature, four pancreatic solid-cystic desmoid tumors have been reported, although pancreatic desmoid tumors are extremely rare (Table 1) [6,8-10]. In this case, the tumor originated in the stomach and infiltrated the adjacent pancreatic body as confirmed pathologically. It is possible that desmoid tumors at pancreatic lesions are more frequently related to cystic changes; however, further accumulation of cases is warranted to explain this cystic change.

The mesentery and retroperitoneum are common anatomic locations of intraabdominal desmoid tumors. In the current case, the tumor originated from the stomach, and locally invaded the retroperitoneum and pancreas body, based on the histopathology of the resected specimen. Desmoid tumors can theoretically arise from any part of the body, but those arising from the stomach are extremely rare [12]; thus, mortality is unknown. Desmoid tumors of the remnant stomach may be associated with surgical trauma from a previous gastrectomy [13].
To the best of our knowledge, this is the first report of a desmoid tumor of the stomach with cystic change that originated in a patient with no history of FAP or surgical trauma.

Intraabdominal desmoid tumors rarely metastasize, but may show local invasion and recurrence [14]. The recurrence rate for desmoid tumors is reported to be $30-40 \%$, but that of sporadic desmoid tumors is lower than in patients with FAP [14]. Therefore, complete surgical resection with negative margins is required to achieve curability. We performed a laparoscopic spleenpreserving distal pancreatectomy (SPDP) combined resection of the gastric wall. Considering the oncologic prognosis of desmoid tumor, the surgical margin must be secured to achieve complete resection, but neither lymph node dissection nor wide resection were needed. We chose distal pancreatectomy to avoid the risk of histologically incomplete resection by the extirpation of the tumor. SPDP is beneficial for patients, because it can prevent an increased risk of overwhelming postsplenectomy infection by preserving the splenic immunological function [15]. Laparoscopic surgery is desirable not only because it is minimally invasive, but also because it favors spleen preservation due to a magnified laparoscopic view, which allows for safe dissection of the small pancreatic vessels [16].

In conclusion, we encountered an extremely rare cystic desmoid tumor at a gastro-pancreatic retroperitoneal site that was difficult to diagnose preoperatively. Laparoscopic SPDP with wedge resection of the stomach was performed safely; such procedures could offer an optimal method to achieve both curability and preservation of function.

\section{Abbreviations \\ CT: Computed tomography; EUS: Endoscopic ultrasonography; FAP: Familial adenomatous polyposis; SPDP: Spleen-preserving distal pancreatectomy}

\section{Acknowledgements}

The authors thank Ms. A. Ohyama and Ms. E. Miyamoto for technical assistance.

Authors' contributions

$\mathrm{KS}, \mathrm{TI}, \mathrm{MO}, \mathrm{YM}$ and $\mathrm{TH}$ collected, analyzed and interpreted the patient data. $\mathrm{RY}$ and KT performed and interpreted the histological examination. KS, MI, 
MM and $Y T$ were responsible for study design, writing and scientific revision. All authors read and approved the final manuscript.

\section{Funding}

None.

\section{Availability of data and materials}

Not applicable.

\section{Ethics approval and consent to participate}

The study was approved on institutional ethical review board of National Hospital Organization Kyushu Cancer Center (reference number: 19-054).

\section{Consent for publication}

The patient provided written informed consent for the publication. The patient's anonymity was preserved.

\section{Competing interests}

The authors declare that they have no competing interests.

\section{Author details}

'Department of Hepatobiliary-Pancreatic Surgery, National Hospital Organization Kyushu Cancer Center, 3-1-1 Notame, Minami-ku, Fukuoka 811-1395, Japan. ²Department of Gastroenterological Surgery, National Hospital Organization Kyushu Cancer Center, Fukuoka, Japan. ${ }^{3}$ Department of Hepato-Biliary-Pancreatology, National Hospital Organization Kyushu Cancer Center, Fukuoka, Japan. ${ }^{4}$ Department of Orthopedic Surgery, National Hospital Organization Kyushu Cancer Center, Fukuoka, Japan. ${ }^{5}$ Department of Pathology, National Hospital Organization Kyushu Cancer Center, Fukuoka, Japan

\section{Received: 26 October 2019 Accepted: 29 January 2020}

\section{0.}

\section{References}

1. Reitamo JJ, Hayry P, Nykyri E, Saxen E. The desmoid tumor. I. Incidence, sex-, age- and anatomical distribution in the Finnish population. Am J Clin Pathol. 1982;77(6):665-73.

2. Gurbuz AK, Giardiello FM, Petersen GM, Krush AJ, Offerhaus GJ, Booker SV, et al. Desmoid tumours in familial adenomatous polyposis. Gut. 1994:35(3):377-81.

3. Dinauer PA, Brixey CJ, Moncur JT, Fanburg-Smith JC, Murphey MD Pathologic and MR imaging features of benign fibrous soft-tissue tumors in adults. Radiographics. 2007;27(1):173-87.

4. Cholongitas E, Koulenti D, Panetsos G, Kafiri G, Tzirakis E, Thalasinou P, et al. Desmoid tumor presenting as intra-abdominal abscess. Dig Dis Sci. 2006;51(1):68-9.

5. Ko SF, Lin JW, Ng SH, Huang CC, Wan YL, Huang HY, et al. Spontaneous isolated mesenteric fibromatosis: sonographic and computed tomographic findings with pathologic correlation. Ultrasound Med Biol. 2006;32(8):1141-9.

6. Amiot A, Dokmak S, Sauvanet A, Vilgrain V, Bringuier PP, Scoazec JY, et al. Sporadic desmoid tumor. An exceptional cause of cystic pancreatic lesion. JOP. 2008;9(3):339-45.

7. Tan $\mathrm{CH}, \mathrm{Pua} \mathrm{U}$, Liau KH, Lee HY. Mesenteric desmoid tumour masquerading as a fat-containing cystic mass. Br J Radiol. 2010;83(994):e200-3.

8. Xu B, Zhu LH, Wu JG, Wang XF, Matro E, Ni JJ. Pancreatic solid cystic desmoid tumor: case report and literature review. World J Gastroenterol. 2013;19(46):8793-8.

9. Mourra N, Ghorra C, Arrive L. An unusual solid and cystic pancreatic tumor in a 20-year-old woman. Desmoid Tumor. 2015;149(3):e5-6.

10. Patel HD, Desai NR, Som A, Shah SK, Thosani NC. Solid-cystic pancreatic tail Desmoid tumor with Beta-catenin positivity. ACG Case Rep J. 2017:4:e40.

11. Lee KC, Lee J, Kim BH, Kim KA, Park CM. Desmoid-type fibromatosis mimicking cystic retroperitoneal mass: case report and literature review. BMC Med Imaging. 2018;18(1):29.

12. Miyata K, Fukaya M, Nagino M. Gradually shrinking intra-abdominal desmoid tumor derived from the stomach in a young boy: a case report. Surg Case Rep. 2017;3(1):54.

13. Date K, Shima Y, Okabayashi T, Iwata J, Sumiyoshi T, Kozuki A. Desmoid tumor of the stomach. Endoscopy. 2015;47(Suppl 1):UCTN:E242-3.
14. Ballo MT, Zagars GK, Pollack A, Pisters PW, Pollack RA. Desmoid tumor: prognostic factors and outcome after surgery, radiation therapy, or combined surgery and radiation therapy. J Clin Oncol. 1999;17(1):158-67.

15. Malleo G, Damoli I, Marchegiani G, Esposito A, Marchese T, Salvia R, et al. Laparoscopic distal pancreatectomy: analysis of trends in surgical techniques, patient selection, and outcomes. Surg Endosc. 2015;29(7):1952-62.

16. Nakata K, Shikata S, Ohtsuka T, Ukai T, Miyasaka Y, Mori Y, et al. Minimally invasive preservation versus splenectomy during distal pancreatectomy: a systematic review and meta-analysis. J Hepatobiliary Pancreat Sci. 2018; 25(11):476-88.

\section{Publisher's Note}

Springer Nature remains neutral with regard to jurisdictional claims in published maps and institutional affiliations.
Ready to submit your research? Choose BMC and benefit from:

- fast, convenient online submission

- thorough peer review by experienced researchers in your field

- rapid publication on acceptance

- support for research data, including large and complex data types

- gold Open Access which fosters wider collaboration and increased citations

- maximum visibility for your research: over $100 \mathrm{M}$ website views per year

At $\mathrm{BMC}$, research is always in progress.

Learn more biomedcentral.com/submission 